\title{
ESTAGEST: an obstetrical application program in BASIC for the computation of estimated date of confinement
}

\author{
H. Frank Andersen and Mel L. Barclay \\ The Department of Obstetrics and Gynecology, The University of Michigan, Ann Arbor, MI, U.S.A.
}

\begin{abstract}
The establishment of the estimated date of confinement (EDC) is an essential element in the care of pregnant women. Unfortunately, accurate information on the last menstrual period, the usual determinant of EDC, is not always available, or clinical estimations of gestational age may be discrepant. Prior studies have demonstrated that an average of several clinical examinations can provide a useful prediction of EDC. We have developed a simple BASIC computer program which allows the clinician to input up to 20 clinical examinations and determines the average EDC based on the clinical dates. The program displays the EDC for each individual examination, which provides an indication of the dispersion, or discrepancy, among the examinations. Average intervals to delivery date from the occurrence of clinical estimators of gestation are based on observed data at our institution and can be easily modified to fit local norms.
\end{abstract}

Obstetrics; Delivery date; Microcomputer; Date averaging; Prediction; Artificial intelligence

\section{Introduction}

One of the essential elements in the antenatal care of pregnant women is the establishment of their estimated date of delivery or confinement (EDC). The classical method of making this determination in clinical practice is Naegele's Rule. According to this method, one substracts three months and adds seven days to the date of onset of the last menstrual period (LMP). This method is rapid, easily performed, and provides an estimate which places the EDC 280 to 284 days from the date of the LMP.

There are, however, a large number of clinical situations in which the LMP is unknown or uncertain. Additionally, an accurately projected delivery date is particularly important in pregnancies

\footnotetext{
Correspondence: H.F. Andersen, Department of Obstetrics and Gynecology, L3221 Women's Hospital, Box 0264, The University of Michigan Medical Center, Ann Arbor, MI 48109-0264, U.S.A.
}

complicated by medical problems such as maternal diabetes, pregnancy-induced hypertension, and erythroblastosis, all of which require well-timed delivery in order to insure maximum fetal survival.

\section{Background}

A number of clinical examinations have been used as estimators of gestational age in the human $[1,2]$. Among those estimators used frequently by the clinician are: (1) LMP, (2) appearance of fetal heart tones audible with an unamplified fetoscope, (3) uterine fundus at the pelvic brim or at the umbilicus, (4) sonographic examination of the fetus, and (5) quickening, the mother's first perception of the baby's movements.

Since all the methods of estimation involve some error, the combined information may be divergent and confusing [1-4]. However, the average of several clinical measures may be useful in predicting term delivery date [2]. Additionally, a 
TABLE 1

Clinical examinations: mean interval to delivery in days [1]

\begin{tabular}{lrll}
\hline Obstetrical milestone & $n$ & $\begin{array}{l}\text { Mean } \\
\text { interval }\end{array}$ & S.D. \\
\hline Last menstrual & 297 & 284.2 & 14.6 \\
Quickening in primipara & 119 & 152.8 & 17.5 \\
Quickening in multipara & 85 & 161.4 & 17.6 \\
Fetal heart audible & 182 & 136.2 & 17.0 \\
Uterus at umbilicus & 95 & 140.8 & 14.9 \\
\hline
\end{tabular}

measure of the statistical dispersion of the various clinical measurements may be useful in evaluating the array of clinical data.

The purposes of the program described here are twofold:

(1) To provide a computerized technique for establishment of a projected EDC given relevant clinical examinations.

(2) To provide information regarding the dispersion of projected delivery dates based on the various clinical examinations.

Each of the estimators specified above are associated with an average remaining duration of gestation, and thereby a projection of EDC. In work reported elswhere, constants have been developed which provide an EDC projection given the date of the occurrence of certain pregnancy-related events or measurements [1]. These constants are listed in Table 1.

\section{Materials and methods}

This program was originally developed in North Star BASIC, and has been modified for IBM PC BASIC. The source listing is compatible with most dialects of BASIC.

\section{Algorithm}

The predicted EDC from combined clinical estimators is obtained by calculating the average EDC based on the clinical estimators. Since each clinical estimator has a different level of accuracy for predicting the EDC a weighted average, allowing more influence for more accurate predictors would seem to be a preferable approach. However, earlier research has suggested that the simple average is as accurate in predicting the EDC as a weighted average [2].

The average EDC is calculated from the predicted intervals to delivery based on an arbitrary reference date. The computational tasks involved in this method are counting calendar days and calculating calendar dates.

There are numerous methodologies for the calculation of calendar dates and the intervals between two dates [5-8]. The Julian method, where days are numbered sequentially from a standard reference date (1 January 4713 B.C.), is not wellsuited to microcomputers since the computations may occur at the limits of precision of 8- to 10-place integer arithmetic [6]. Although Julian techniques can be modified to operate on microcomputer systems, an alternative system was utilized. The system used here is based on the sequential day number in the year (modulo date). It was chosen because it presents the dates in a convenient way and circumvents problems with computational accuracy. A complete description of this methodology may be found in Andersen's paper [5]. Two subroutines in the program convert a date in month/day/year format into a modulo date and convert a modulo date back to month/ day/year format. Specific subroutines convert calendar date into modulo date, modulo date back to calendar date, calculate the interval between two dates in modulo format, and add a specific interval in days to a modulo date.

\section{Program description}

The program is menu-driven and allows the user to choose any of a list of gestational estimators. In addition to gestational estimators with a specific, average interval to EDC (e.g., fetal heart tones first audible or quickening), the user may enter other gestational estimators (e.g., fundal height measurement) by entering the date of the measurement and the estimated gestational age by that measurement. After all of the relevant estimators are entered, the user chooses the final option, 
'compute average EDC', which causes the printing of a summary table and the termination of the program.

Typing errors and inappropriate data entry must be corrected within the standard BASIC input procedures. The data must be entered in uniform fashion, that is, month, day, year form. The year may be entered as 19XX providing all subsequent entries are made in a similar fashion. This program does not provide for editing the list of data after entry.

Following data entry and assignment of variable names, the data are stored and converted from calendar-date form to modulo-date form. The estimated date of confinement associated with a

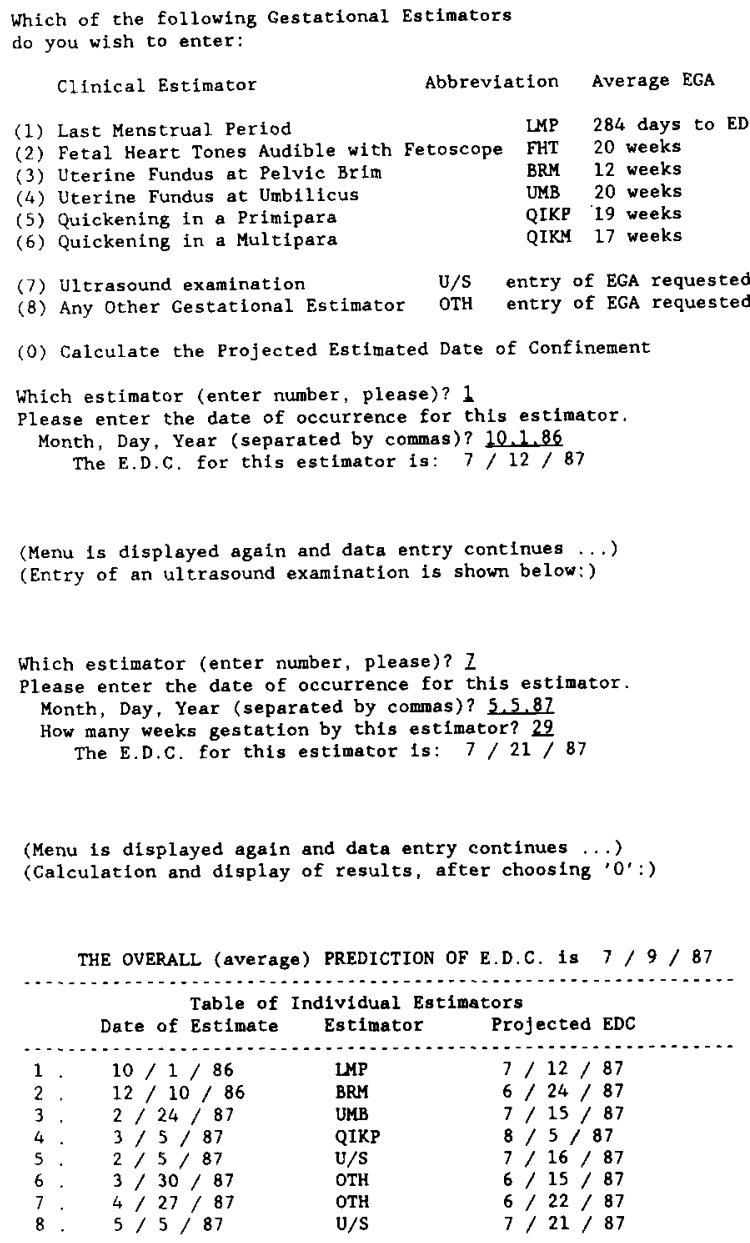

Fig. 1. Computer output of a sample patient run. (User input is underlined.) particular date and clinical estimator is calculated by the addition of the appropriate number of days. The table of projected intervals from the gestational estimator to term delivery date can be modified according to local findings, if necessary. The interval from the reference date to the projected EDC is calculated and stored in the result matrix.

A sample output for a patient is shown in Fig. 1.

An annotated program listing is included in the appendix. The program is listed in structured format with variable definitions, data types, and constants specified. All subroutines are labelled.

\section{Discussion}

A number of schemata are available for the statistical manipulation of EDC related data. Many relate to the issue of the uncertainty of projected EDCs [1-3]. In previous studies where the data was incomplete or conflicting, analytic methods using multiple linear regression techniques or graphic methodologies were found to be no more accurate than the simple arithmetic mean in the projection of EDC [2-4].

Computerized analysis of this type of calendar data also provides the clinician with a measurement of the dispersion of clinical estimators of gestational age. In this program we display predicted EDCs for each individual examination to provide the clinician with an indication of that dispersion. At present the significance of discrepancies among clinical estimators in relation to confidence intervals for predicting EDC is not known. Further research may allow calculation of confidence intervals for these estimates. This information may be factored into decisions regarding the value of a particular intervention as well as its relative risk.

\section{References}

[1] H.F. Andersen, T.R. Johnson Jr., M.L. Barclay and J.D. Flora $\mathrm{Jr}$., Gestational age assessment. I. Analysis of individual clinical observations, Am. J. Obstet. Gynecol. 139 (1981) 173. 
[2] H.F. Andersen, T.R. Johnson Jr., J.D. FLora Jr. and M.L. Barclay, Gestational age assessment. II. Prediction from combined clinical observations, Am. J. Obstet. Gynecol. 140 (1981) 770.

[3] T.R. Johnson Jr. and B.A. Work Jr., A dynamic graph for documentation of gestational age, Obstet. Gynecol. 54 (1979) 115.

[4] W.J. Ott, Accurate gestational dating, Obstet. Gynecol. 66 (1985) 311.

[5] H.F. Andersen, Calendar: a BASIC subroutine to manipulate calendar dates, Recreational Computing 8/3 (1979) 25.

[6] G. King, Julian dates for microcomputers, Dr. Dobbs J. 8/6 (June 1983) 66

[7] R.G. Tantzen, The Collected Algorithms of the ACM (1963).

[8] S.G. Levy, BASIC dates (letter), Dr. Dobbs J. 9/1 (January 1984) 10.

\section{Appendix}

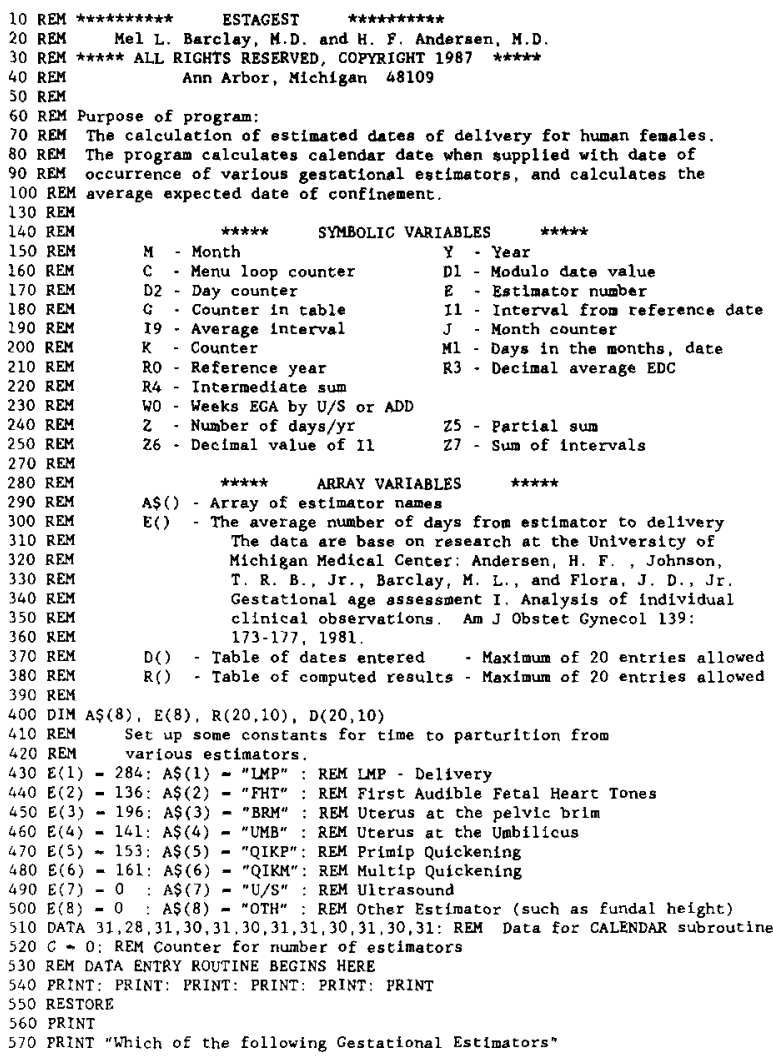

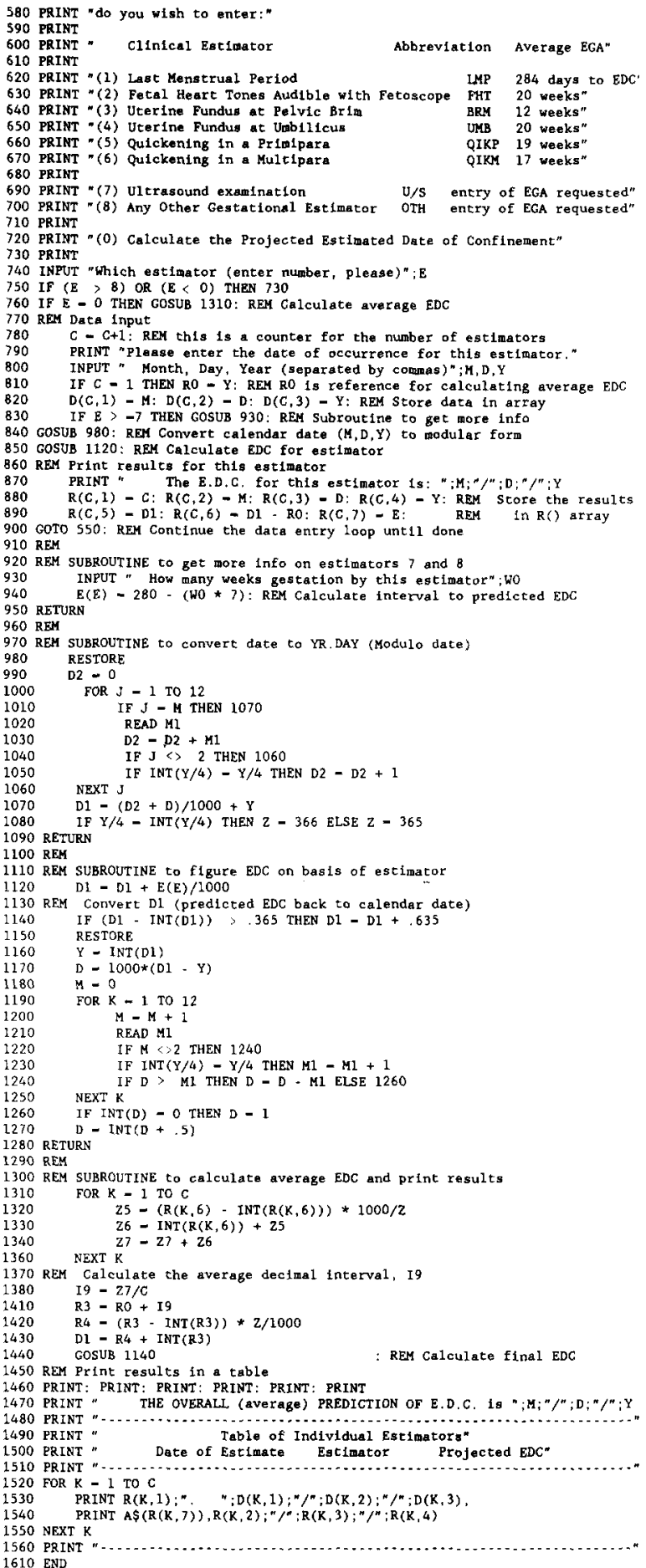

